\title{
GAUCHER'S DISEASE IN BONE
}

\author{
F. W. T. Davies, Burton-on-Trent, England
}

The first complete radiological investigation of Gaucher's disease in bone has been attributed to Junghagen in 1926. Pick (1933) gave the classical account of the clinical features of the disease, and most authors have followed his conceptions more or less closely, with a few dissenters. In his paper, Pick distinguished an " osseous type " with predominantly an osseous distribution, stating that " scarcely a bone is spared." He suggested a constitutional predisposition and supported this contention with an example of a whole generation who exhibited osseous lesions in those members affected. More recently a familial incidence has been recorded in which a non-osseous lesion predominated (Morgans 1947). Many case records in the literature have shown the disease to have commenced with splenomegaly and to have developed osseous lesions later. Indeed, so often has this order of progress of the disease been encountered that some writers have suggested that spread to the skeleton has been precipitated by splenectomy. Others have maintained that splenectomy increases resistance to acute infections and therefore is a desirable form of treatment (Logan 1941).

According to Pick, the typical lesion occurring in the femoral head consisted of " an invasion of the femoral head with subsequent collapse and deformity, leading to a deforming arthritis." Reed and Sosman (1942) have supported this finding and describe a " pathological fracture occurring in the destructive types of lesion." They state that the deformity occurring in hip lesions is to be distinguished from Perthes' disease, tuberculous disease and chronic osteomyelitis.

Microscopic examinations made by Pick showed the marrow to be interspersed with nodules of varying size, partly encapsulated, packed with cells of typical type and containing the distinct staining characteristics associated with the more usual non-osseous lesions. Cushing and Stout (1926) demonstrated the presence of large cells in the marrow of the femoral head and neck, producing an "osteo-arthritis with sequestrum formation." They also observed that the cells found in the bone marrow frequently assumed a spindle formation which was thought to be the result of mutual compression. It is possible that such compression may influence adversely the vascularity of the bone adjacent to the infiltrated areas. More recently Schein and Arkin (1942) have suggested that vascular obstruction was due to direct infiltration of the vessels by Gaucher's cells. In early descriptions many patients were wrongly described as suffering from osteomyelitis, and it may be that true sequestrum formation was the direct outcome of surgical drainage.

Other general features common to all forms of Gaucher's disease include patchy pigmentation of the skin and deposits occurring in the sclera, known as pingueculae. A haemorrhagic diathesis is also alleged to occur.

The following case report illustrates apparent resolution of the disease in one area, while further spread occurred in other zones of the affected bone and in other parts of the skeleton. A note is added on the value of deep $x$-ray therapy.

\section{GASE REPORT}

A young unmarried woman aged nineteen years gave a history of pain and limp affecting the right hip for the previous six months. There was a history of splenectomy four years before, undertaken because of pain in the left hypochondrium associated with splenomegaly. At that time blood counts, sternal puncture and skin biopsy failed to show any evidence of infiltration by Gaucher's cells. Examination of the spleen, however, confirmed the diagnosis 


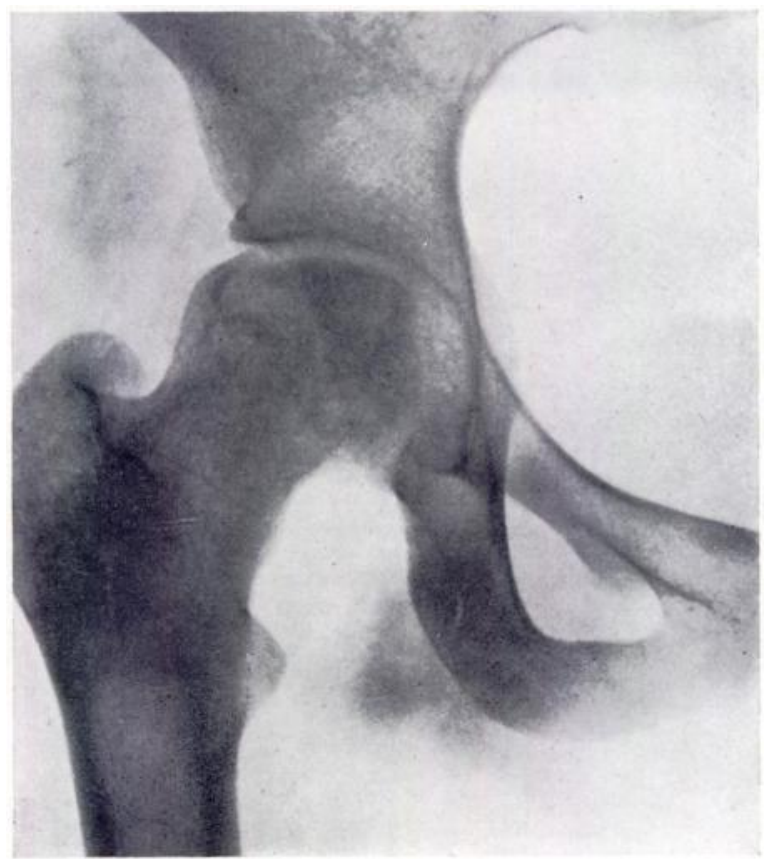

FIG. 1

Right hip at time of admission at age of nineteen years. Note depressed avascular fragment at upper segment of femoral head.

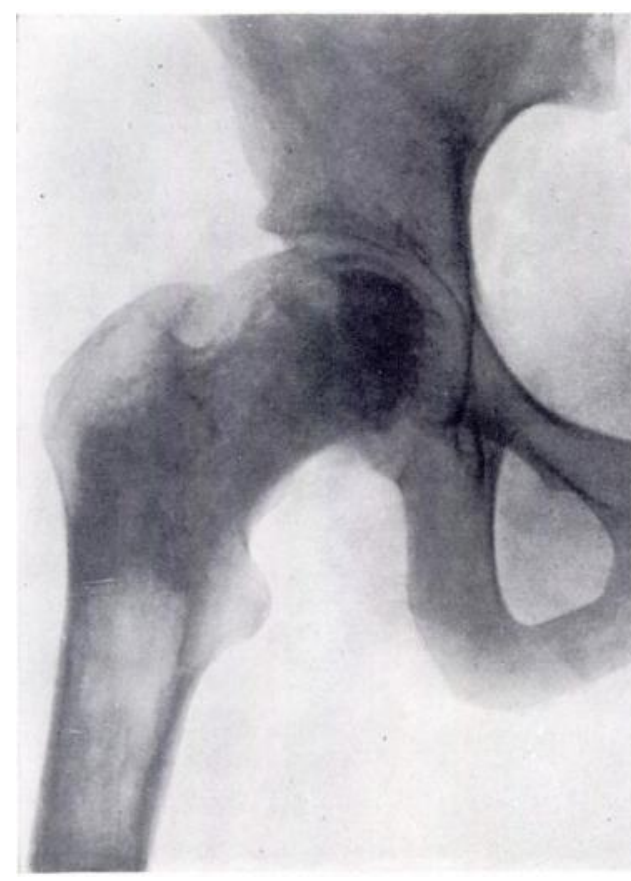

FIG. 2

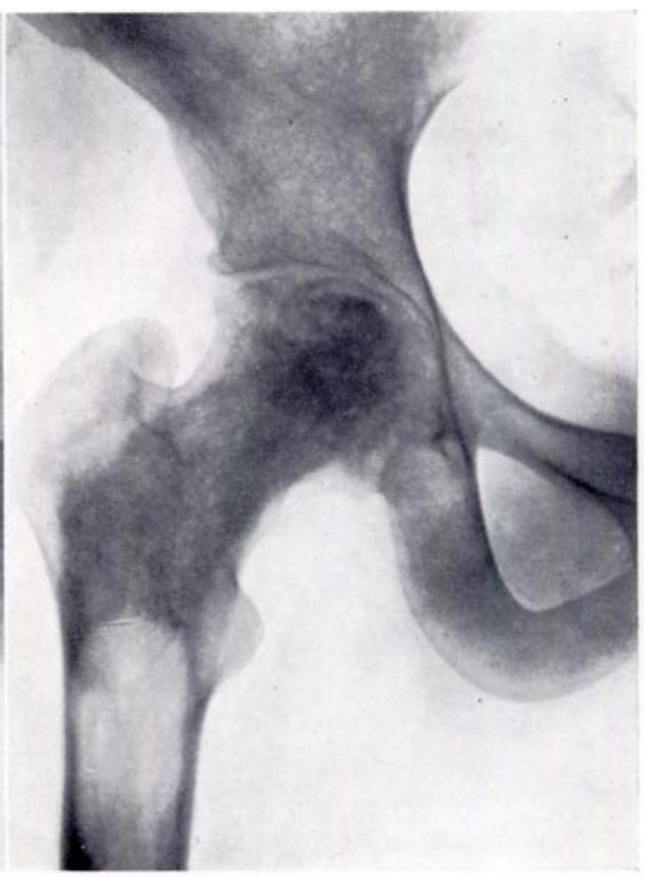

FIG. 3

Figure 2-Three months later. In addition to the changes in the femoral head an early crstic appearance is shown in the shaft. Figure 3-Three months later still. Note absorption of avascular fragment.

vol. $34 \mathrm{~B}$, NO. 3, AUGUST 1952

$\mathrm{H}_{14}$ 
of Gaucher's disease. At that time no radiographic investigation of the skeleton had been conducted.

On examination there was apparent shortening of the right lower limb, a slight flexion contracture of the right hip and marked limitation of all movements due to pain. Radiographs showed changes suggestive of a form of osteochondritis of the right femoral head (Fig. 1), together with evidence of cavitation in the upper extremities of the shafts of both femora. Treatment-Traction was applied to both lower limbs and maintained for nine months, during which there was progressive improvement in the radiographic appearance of the femoral head. The previously arascular segment in the upper half of the head appeared to become gradually revascularised (Figs. 2 and 3). The hip was subsequently immobilised in a plaster spica for seven months, after which she was discharged home wearing a short spica.

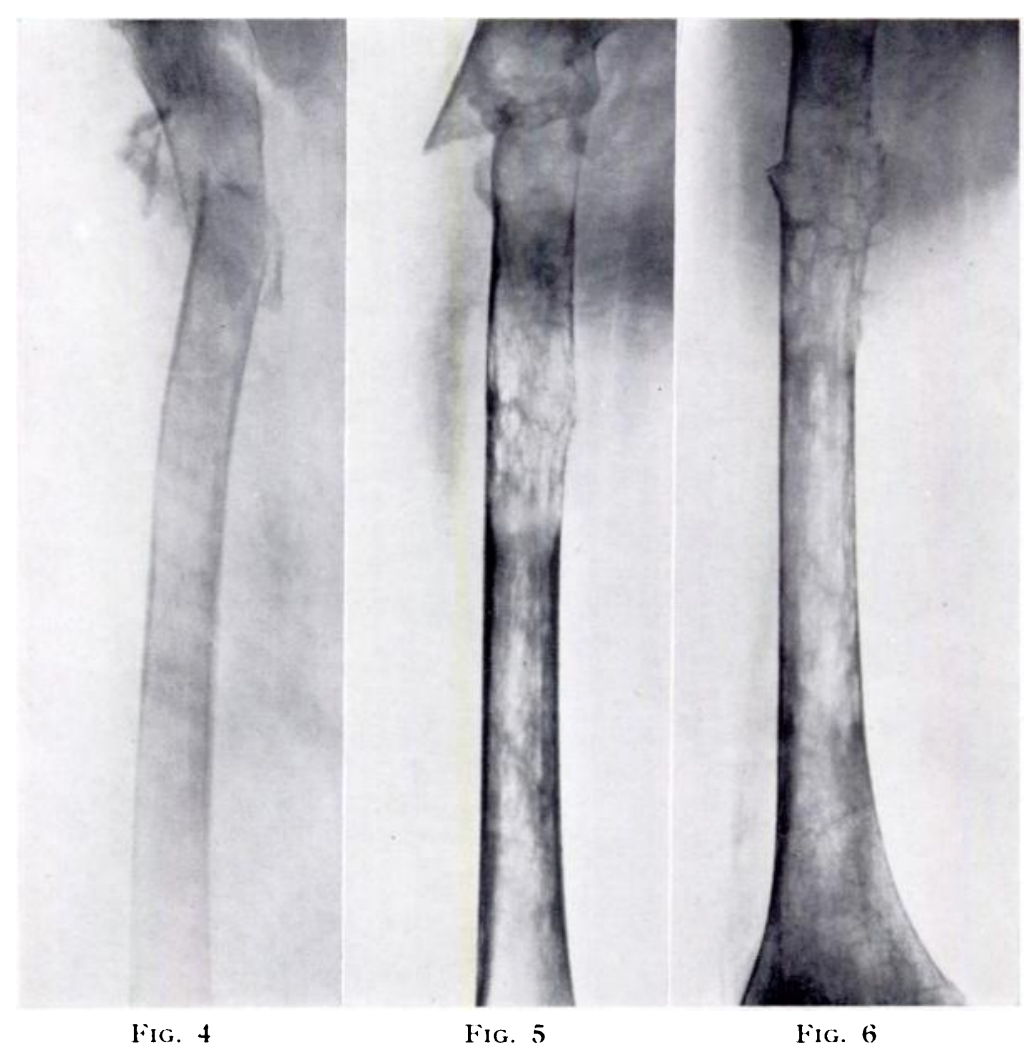

Figure 4--Right hip showing pathological fracture through cystic area at upper end of shaft. Figures 5 and 6-Progressive changes in the femoral shaft below the pathological fracture. Figure 5 shows the condition four months after the fracture. Figure 6-live months later.

Progress-The patient later sustained a pathological fracture through a cystic area in the upper end of the right femoral shaft (Fig. 4). Traction was reapplied and immobilisation resumed in recumbency. Radiographic studies were continued during the following months. Progressive destruction of the cortex of the shaft of the right femur was observed below the level of the fracture (Figs. 5 and 6 ). In the left femur infiltration of the marrow cavity was seen to continue (Figs. 10 and 11), but progressed less rapidly than the changes in the right side. During these studies it was observed that the characteristic flask-like enlargement of the lower femoral shaft (Fisscher's sign) did not occur in this patient, in spite of the severity of the lesion higher up the shaft. Biopsy was performed, scrapings being obtained both from 
the base of the neck of the femur and from the upper cystic segment of the shaft. Both specimens were reported to contain massive infiltration by Gaucher's cells, in spite of apparent resolution as seen radiographically.

Response to $x$-ray therapy-It has been commonly accepted that Gaucher's disease is unaffected by deep $\mathrm{x}$-ray therapy. In this instance, however, it was decided to give this form of treatment a trial. The response was striking, especially in areas of the right femur in which cortical destruction had occurred (Figs. 7 to 9). Later radiographs suggested that the process had been halted and that there had been an actual increase in the number, dimensions and density of the trabeculae (Fig. 9). The total irradiation given was 2,500 and represents the estimated tumour dose at the centre of the limb. Treatment for the left femur was begun later and the response was satisfactory (Figs. 11 and 12).

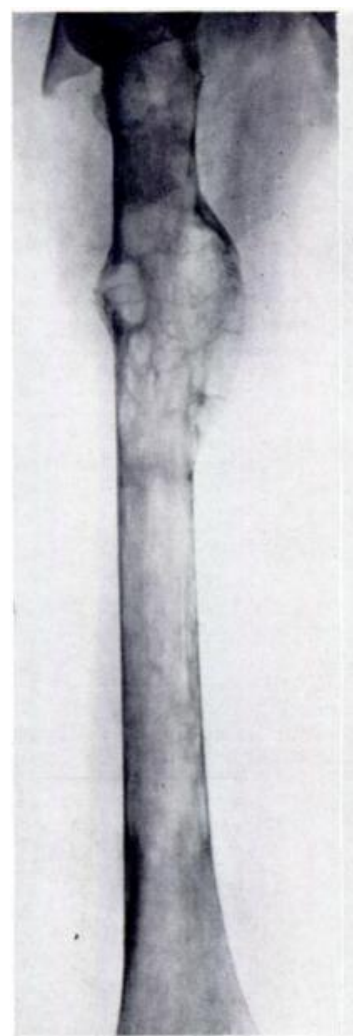

FIG. 7

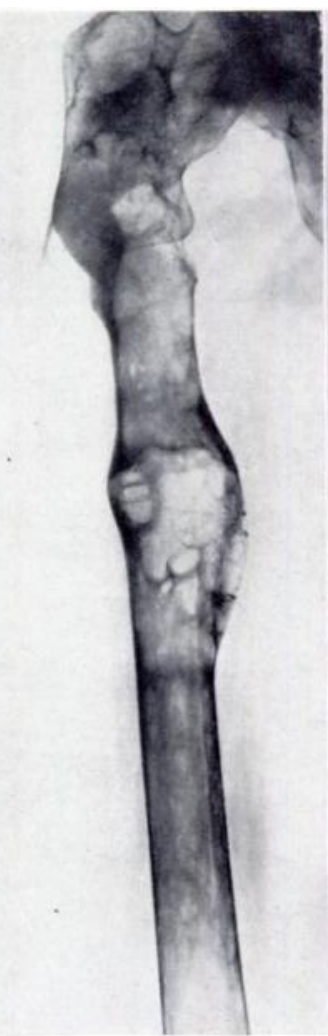

FIG. 8

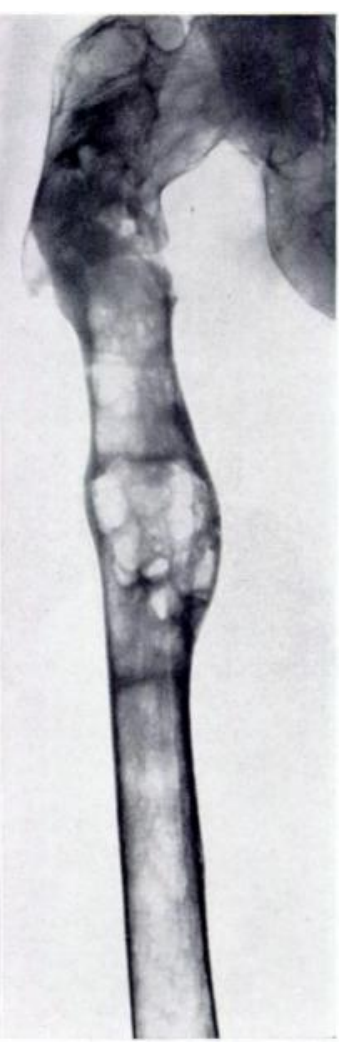

FIG. 9

Right femur showing response to radiation therapy. Figure $7-$ Two months after radiation (seven months after radiograph shown in Figure 5). Figure 8One and a half years after radiation therapy. Figure 9 -Three and a half years after radiation.

\section{DISCUSSION}

This case presents several unusual features. At the time of splenectomy and possibly before any appreciable osseous involvement had occurred, no deposits of cerebroside were found in the skin biopsy from areas of doubtful pigmentation. Sternal puncture was at that time negative. Regression of the process occurred in one area of an affected bone, while progressive infiltration occurred elsewhere in the same bone and in other parts of the skeleton.

The writer considers that the progressive absorption of the avascular fragment of the femoral head could not have occurred if the blood vessels were infiltrated with Gaucher's cells as suggested by Schein and Arkin (1942). Biopsy, performed after absorption was

vol. 34 B, No. 3, AUGUST 1952

$\mathrm{HI}_{(4)}$ 


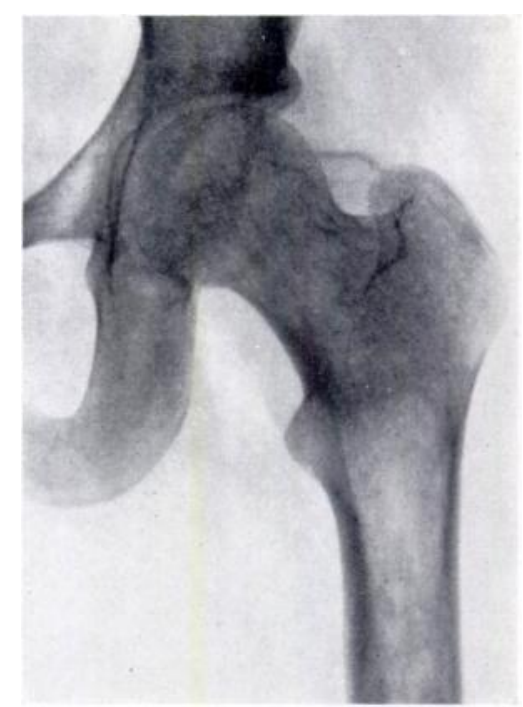

Fig. 10

Left femur at age nineteen, shortly. after admission. Early cystic appearance present in upper part of the femoral shaft.

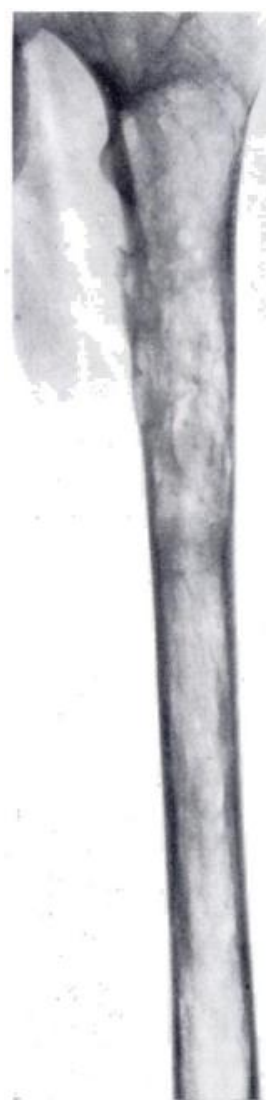

FIG. 11

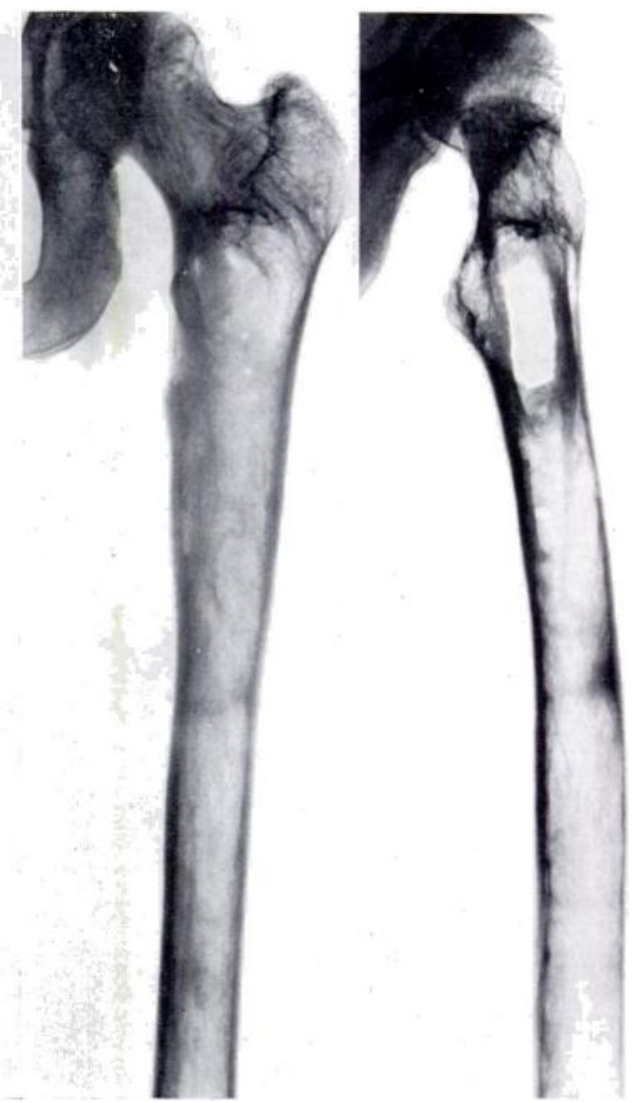

FIG. 12

Figure 11-Two years later: changes more marked, but less severe than in right femur. Figure 12-Four years later still, showing condition two years after treatment by radiation.

THE JOURNAL OF BONE AND JOINT SURGERY 
complete, confirmed intensive infiltration of the femoral neck with Gaucher's cells. It is possible that if weight-bearing and muscle pull are eliminated, an adequate blood supply may be restored ultimately.

Deep x-ray therapy appears to afford some hope of success in arresting the progressive infiltration of bone, and there is evidence to suggest that increased trabeculation can be induced. Contrast of the early radiographs with the most recent films taken six years after the condition was diagnosed and three and a half years after treatment by deep x-ray therapy, show many interesting features (Figs. 7 to 9 and Fig. 12). There appears to have been a cessation of the process of cortical disruption in the shaft of the right femur. The outline of the femoral head has regained a reasonable shape. No further extension of the medullary cavitation of the left femur occurred and in fact the writer considers that there has been some regression and retrabeculation after deep $\mathrm{x}$-ray therapy.

Although this patient has been examined at regular intervals over the past six years, no further spread has been found in other parts of the skeleton. It may be that the pathological process has been arrested: certainly there seems to be no doubt that it has been slowed very appreciably, when one remembers that most of the infiltration appears to have occurred in the first three years that the patient was under observation.

It may be argued that retrogression was already occurring in the first lesion in the femoral head before irradiation was begun. Nevertheless, the recent films do not indicate any evidence of the " deforming arthritis" to which Pick (1933) referred in his account, and which appears to be the usual outcome in untreated cases.

I am indebted to Mr A. M. Hendry of Birmingham for permission to publish this case and for the interest which he has taken, and to Sir Reginald Watson-Jones for his encouragement.

\section{REFERENCES}

Cushing, E. H., and Stout, A. P. (1926): Gaucher's Disease. With Report of a Case Showing Bone Disintegration and Joint Involvement. Archives of Surgery, 12, 539.

JunghageN, S. (1926): Röntgenologische Skelettveränderungen bei Morbus Gaucher. Acta Radiologica, 5, 506.

Logan, V. W. (1941): The Results of Splenectomy in Gaucher's Disease. Surgery, Gynecology and Obstetrics, 72, 807.

Morgans, M. E. (1947): Gaucher's Disease Without Splenomegaly. Lancet, ii, 576.

Pick, L. (1933): A Classification of the Diseases of Lipoid Metabolism and Gaucher's Disease. American Journal of the Medical Sciences, 185, 453.

Reed, J., and Sosman, M. C. (1942): Gaucher's Disease. Radiology, 38, 579.

Schein, A. J., and Arkin, A. M. (1942): Hip-Joint Involvement in Gaucher's Disease. Journal of Bone and Joint Surgery, 24, 396. 